\title{
Engineered Aerosol Medicine and Drug Delivery Methods for Optimal Respiratory Therapy
}

The human lung is an effective route for noninvasive drug delivery because it provides a large surface area for rapid absorption, a minimal physical barrier, an absence of extreme $\mathrm{pH}$ and metabolism, no first-pass liver, a rich blood supply, and poor bioavailability of oral drugs. The physical and electrical properties of active pharmaceutical ingredient and excipient interactively influence 5 electromechanical deposition mechanisms of respiratory medicinal aerosols while flowing through the airways, including nose, mouth, pharynx, trachea, bronchi, bronchioles, and alveoli.

The therapeutic particles from a specific device during the inhalation process come out as an aerosolized form. The dispersion of these pharmaceutical powders is often difficult because the fine powders are cohesive as a result of the strong interparticle adhesion forces: van der Waals, capillary, and electrostatic attractions. Typically, cohesive forces are proportional to the diameter $d$ of the particles, whereas the detachment forces for resuspending the particles forming aerosol are proportional to $d^{2}$ when aerodynamic shear force is used for the dispersion. Thus, the

See the Original Study on Page 1476

smaller the diameter, the higher the shear force needed for efficient dispersion to form aerosol containing the primary active pharmaceutical ingredient and excipient particles micronized in the jet mill. A solution to these problems can be the manufacture of engineered active pharmaceutical ingredient and excipient to manipulate the cohesivity and dispersibility with necessary magnitudes of particle charge during the micronization process. These modifications will improve blending uniformity and long-term stability of the powder mixtures.

\footnotetext{
The author has disclosed no conflicts of interest.

Correspondence: Mohammed Ali PhD MBA CSMS, Department of Industrial Systems and Technology, Jackson State University, 1400 JR Lynch Street, JSU Box 18480, Jackson, MS 39217. E-mail: mohammed.ali@jsums.edu.
}

DOI: $10.4187 /$ respcare. 03634
The primary mechanisms of lung deposition of inhaled respirable drug aerosol particles in the human lung consist of the 5 electromechanical processes: impaction, diffusion, interception, gravitational settling, and electrostatic effects, as depicted in Figure 1.1,2 There are a number of mathematical models and computational tools developed to understand particle flow and to predict deposition patterns of particles in the whole lung or its specific region. ${ }^{3-9}$ In silico study results using these modeling tools agreed with in vitro study results as well. ${ }^{10,11}$

In the field of aerosol medicine, there have been several methods invented and marketed to improve pharmacokinetic efficacy and effectiveness in respiratory drug delivery, and to minimize losses in the delivery devices. Examples are: nanoparticle therapeutics for reduced side effects and more targeted deposition, salt-based formulations optimized for inhalation to create a robust and flexible platform that can accommodate low or high drug loads of a range of molecule types, sustainable long pulmonary absorption achieved through tissue binding and cellular uptake in the airways, moderately lipophilic compounds with positive electrostatic charge under physiological conditions to bind preferentially lung tissues, liposomal encapsulation in controlled-release particles, and increasing the molecular mass of the drug by conjugation with a water-soluble inert ligand or another protein. ${ }^{12-14}$

Additional examples of improved drug delivery methods include: the use of a valve holding chamber or spacer for metered-dose inhalers; inlet design change of dry powder inhalers for less resistance to air flow and particle de-agglomeration; face mask with nebulizer and spacer for infants and children; charge neutralization of nebulized aerosol before administration; nonpropellant inhaler with soft mist of aqueous drug solution through a set of fine channels in the nozzle; enhanced condensational growth of submicron particles for minimum mouth-throat deposition; and evaporation and condensation aerosol generator for poorly water-soluble drugs suitable for pulmonary route. ${ }^{1,15,16}$ Studies with laboratory animals showed that the required antibiotic dose of nanoaerosol generated by electrospray-neutralization process for the pulmonary drug delivery method is one-sixth of the dose for peroral treatment. ${ }^{15,17}$ 


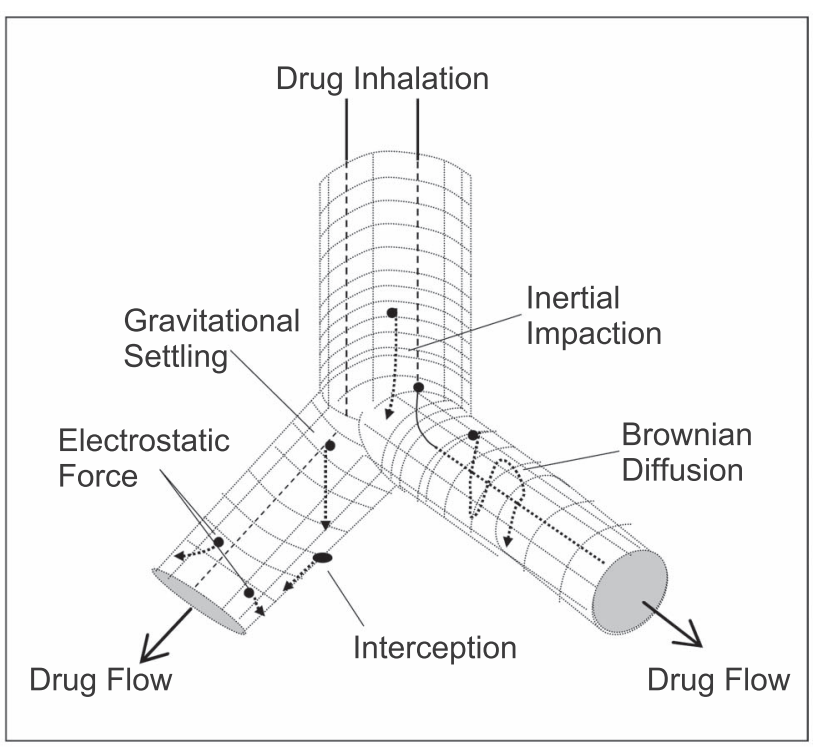

Fig. 1. Respiratory drug particle deposition mechanisms in the human lung. From Reference 1, with permission.

High-flow nasal cannula therapy is currently being employed to deliver oxygen to patients with moderate levels of hypoxemic respiratory problems..$^{18}$ In the same fashion, this method can be used to deliver drugs to the lungs. However, it was reported that infant, pediatric, and adult nasal cannulas show a decrease in delivery with an increase in inspiratory flow, which can be explained by the higher drug deposition (losses) in the device components. ${ }^{19-21}$ These losses often occur as a result of combined effects from several issues, such as inside geometry of cannula and directional change of aerosol flow in the nose, mouth, pharynx, and trachea.

The enhanced condensational growth and excipient-enhanced growth showed substantial improvement ( $\sim 80 \%)$ without incorporating exhalation cycle. An in vitro experimental study published in this issue of RESPIRATORY CARE has reported an improved respiratory drug delivery method by employing intermittent aerosol delivery during highflow nasal cannula therapy. ${ }^{22}$ Using controlled growth techniques on freshly generated drug aerosols, Golshahi and co-workers ${ }^{22}$ show that the intermittent delivery method provides a better lung dose than the continuous delivery method, by reducing losses in the drug delivery devices. However, significant challenges lie ahead of the success of the application of enhanced condensational growth and excipient-enhanced growth to high-flow nasal cannula therapy, because therapeutic aerosol particle size rapidly changes due to the tendency of hygroscopic growth, and higher mass concentrations are delivered as a consequence. The higher drug particle mass may experience higher inertial impaction and sedimentation deposition in the conducting tracheobronchial airways, which will eventually cause lower amounts of drug delivery and deposition in the respiratory bronchioles and alveoli. The results of growth technique for high-flow nasal cannula therapy may also prove promising if realistic inhalation-exhalation flows are simulated. In addition, well-characterized further experiments are necessary to warrant in vivo studies by noninvasive and pulmonary drug delivery investigators.

Mohammed Ali PhD MBA CSMS

Department of Industrial Systems and Technology Jackson State University Jackson, Mississippi and School of Systems Biology George Mason University Manassas, Virginia

\section{REFERENCES}

1. Ali M. Pulmonary drug delivery. In: Kulkarni K, editor. Handbook of non-invasive drug delivery systems. Amsterdam: Elsevier; 2009: 209-246.

2. Hinds WC. Aerosol technology: properties, behavior, and measurement of airborne particles. New York: Wiley-Interscience; 1999; 235

3. International Commission on Radiological Protection. Human respiratory tract model for radiological protection. New York: Elsevier; 1994.

4. Yeh HC, Schum GM. Models of human lung airways and their application to inhaled particle deposition. Bull Math Biol 1980;42(3): 461-480.

5. Martonen TB, Schroeter JD, Fleming JS. 3D in-silico modeling of the human respiratory system for inhaled drug delivery and imaging analysis. J Pharm Sci 2007;96(3):603-617.

6. Ali M, Reddy RN, Mazumder MK. Electrostatic charge effect on respirable aerosol particle deposition in a cadaver based throat cast replica. J Electrostat 2008;66(7-8):401-406.

7. Finlay WH. Lung deposition simulation. In: Hickey AJ, editor. Pharmaceutical inhalation aerosol technology. New York: Informa; 2004: 155-171.

8. Azhdarzadeha M, Olferta JS, Vehring R, Finlay WH. Effect of induced charge on deposition of uniformly charged particles in a pediatric oral-extrathoracic airway. Aerosol Sci Tech 2014;48(5):508514.

9. Asgharian B, Hofmann W, Bergmann R. Particle deposition in a multiple-path model of the human lung. Aerosol Sci Technol 2001; 34(4):332-339

10. Kleinstreuer C, Zhang Z, Donohue JF. Targeted drug-aerosol delivery in the human respiratory system. Ann Rev Biomed Eng 2008; 10(1):195-220.

11. Cheng YS. Mechanisms of pharmaceutical aerosol deposition in the respiratory tract. AAPS Pharm Sci Tech 2014;15(3):630-640.

12. Patton JS, Byron PR. Inhaling medicines: delivering drugs to the body through the lungs. Nat Rev Drug Discov 2007;6(1):67-74.

13. Davis ME, Chen ZG, Shin DM. Nanoparticle therapeutics: an emerging treatment modality for cancer. Nat Rev Drug Discov 2008;7(9): 771-782.

14. Peng C, Chow AHL, Chan CK. Study of the hygroscopic properties of selected pharmaceutical aerosols using single particle levitation. Pharma Res 2000;17(9):1104-1109. 


\section{Engineered Aerosols and Drug Delivery Methods}

15. Onischuk AA, Tolstikova TG, Sorokina IV, Zhukova NA, Baklanov AM, Karasev VV, et al. Anti-inflammatory effect from indomethacin nanoparticles inhaled by male mice. J Aerosol Med Pulm Drug Deliv 2008;21(3):231-243.

16. Atkins PJ. Next generation technology for pulmonary drug delivery. Drug Deliv Technol 2005;5(6):74-75.

17. Morozov VN, Kanev IL, Mikheev AY, Shlyapnikova EA, Shlyapnikov EM, Nikitin MP, et al. Generation and delivery of nanoaerosols from biological and biologically active substances. J Aerosol Sci 2014;69(3):48-61.

18. Golshahi L, Tian G, Azimi M, Son YJ, Walenga R, Longest PW, Hindle M. The use of condensational growth methods for efficient drug delivery to the lungs during noninvasive ventilation high flow therapy. Pharm Res 2013;30(11):2917-2930.

19. Ari A, Harwood R, Sheard M, Dailey P, Fink JB. In vitro comparison of heliox and oxygen in aerosol delivery using pediatric high flow nasal cannula. Pediatr Pulmonol 2011;46(8):795-801.

20. Rubin BK. Pediatric aerosol therapy: new devices and new drugs. Respir Care 2011;56(9):1411-1421.

21. Dhand R. Aerosol therapy in patients receiving noninvasive positive pressure ventilation. J Aerosol Med Pulm Drug Deliv 2012;25(2):63-78.

22. Golshahi L, Longest PW, Azimi, Syed A, Hindle M. Intermittent aerosol delivery to the lungs during high-flow nasal cannula therapy. Respir Care 2014;59(10):1476-1486. 